\title{
IV. The Method of Serial Groups.
}

BY PROFESSOR GEORGE M. STRATTON.

In the practical conduct of the laboratory one frequently feels the shortcomings of the method of 'minimal changes.' It is undoubtedly the best all-around mode of procedure yet devised, but in certain cases where the conditions are exceptional it may leave one quite im Stich. This is especially true when one is dealing with minimal impressions, where suggestion is apt to find such free play; the observer may continue to notice a sensation when the stimulus has become suspiciously weakin fact when no stimulus at all is applied. Thus, with certain excellent though suggestible subjects, I have found it impossible to determine by the method of minimal changes, pure and simple, the least extent of visible motion that could be perceived as motion. The subjects persisted in seeing the light move on every occasion, whether there was any actual movement or not. The control of the answers, by requiring that the observer shall tell correctly some additional feature of the impression-tell, say, the direction of the movement, or, if the experiments be on the least perceptible change of pressure, tell whether the pressure becomes heavier or lighter - may in some instances be helpful. But often this check will hide the very facts that one wishes to ascertain - the point at which the subject perceives motion and yet is uncertain of the direction, or notice change of pressure without being able to say whether the weight has grown greater or less.

The usual resort in this event is either to a so-called ' catch experiment' the Vexirversuch, where no stimulus at all is given, or to the method of right and wrong cases. The latter, making use as it does of the law of probability, not only requires an extremely large number of observations, but there is usually needed considerable preliminary and irregular experimentation in order to discover the conditions that will give a suitable proportion of right and wrong answers. The Vexirversuch, on the other hand, has never been systematized, and as it is usually introduced in an irregular fashion within the 
method of minimal changes, it is apt to disturb the even tenor of the research, and disconcert the observer whenever he gets a hint of what is being done.

In the method of serial groups here proposed, the attempt is made to legitimate the 'catch' experiment, to introduce it as a continuous and regular element of the procedure, while securing certain advantages both of the method of minimal changes and of the method of right and wrong cases. To give a concrete illustration, suppose the following groups of experiments be carried out to determine the just perceptible extent of movement by sight, under the conditions described in the second part of the preceding paper:

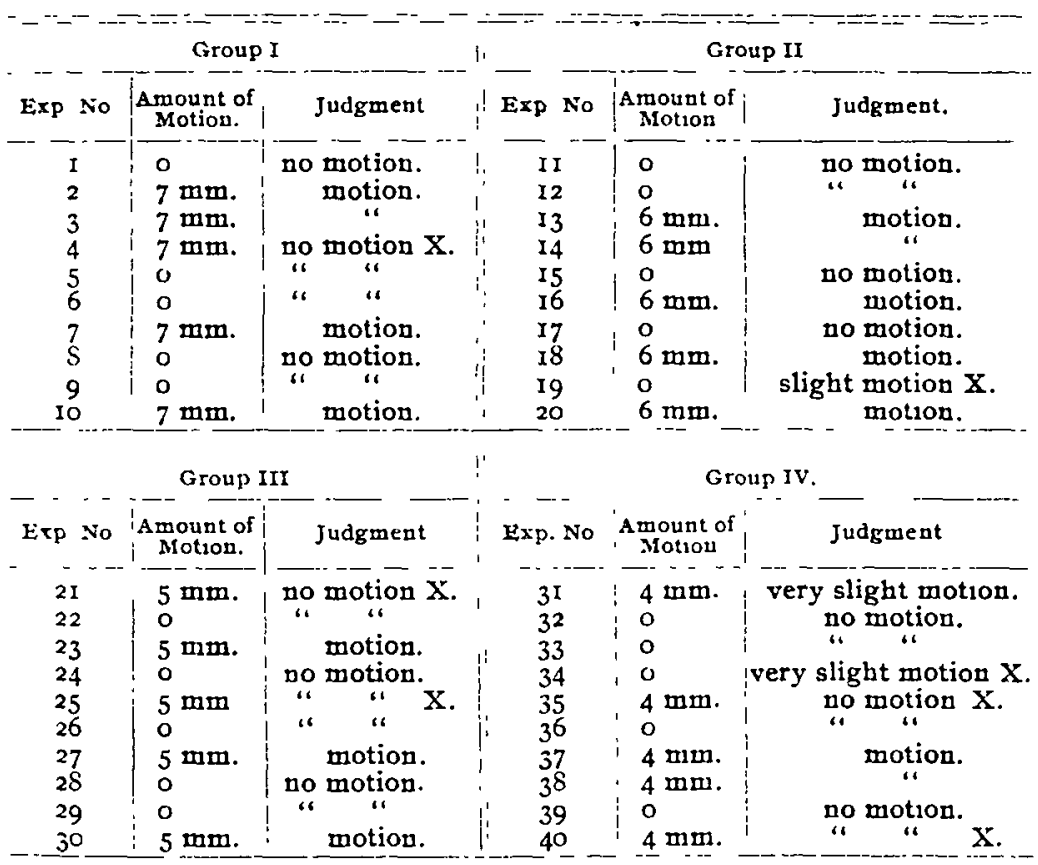

The $\mathrm{X}$ shows the errors in any group, and from these the threshold may be determined according to any proportion of correct and incorrect answers that may be chosen. In my own computations that group has been taken as giving the threshold beyond which less than eight out of the ten judgments are right. But a detail like this, as well as the exact number of 
experiments that may best form a 'group,' might well be considered as subject to revision in the light of farther experience, and not as an essential part of the method. The essence of the matter is simply that there should be groups of experiments arranged in a regular series, the amount of positive stimulus, as one passes from group to group, being graduated according to the principle of the method of minimal changes; while within the limits of any one group a constant stimulus is irregularly alternated with cases where the stimulus is zero, thus uniting in the single group the basal principle of the method of right and wrong cases and that of the Vexirversuch. This may seem provokingly eclectic, but it is not exactly that; the different elements make an organic union, and not a mere patchwork. There is simply an attempt to make systematic what experimenters have frequently found themselves compelled to do in a casual and uncritical way.

One may perhaps repeat that this method is not proposed as a general substitute for the classic ones in use. It is well, however, to multiply our tools so that the best may be selected for the special work in hand. And this one has been found good for certain purposes, especially where suggestion plays a prominent rôle. The observer may here know from the very beginning the general method of procedure; he may know that zerocases are to be irregularly alternated with those of positive stimulation, and his expectation is therefore less 'set' and influential. The zero-cases no longer come in as a kind of indignity upon the observer, as if his word were being questioned. The check here, because of its constancy, ceases to excite any feeling. The procedure, moreover, has the virtue of the method of minimal changes, in that the threshold is ascertained empirically, by actually crossing it. And while the principle of right and wrong cases is employed, with the powerful control which that always brings, yet there is no introduction of the intricate calculus of probability and a certain darkness that always shadows its results. It is true that the application of the method of serial groups is in a certain sense cumbersome, as compared with the method of minimal changes, since in a given time fewer determinations of the threshold can be obtained. But 
with suitable rests between the 'groups,' there is no need of there being greater fatigue to the observer in the one case than in the other; and while the determinations may be fewer for the time expended, yet in most cases I have found that they more than make up in weight what they lack in number.

\section{The Effect of Subdivisions on the Vistal Esty- MATE of TiME.}

\section{BY MABEL LORENA NELSON.}

It has been found by Dr. Ernst Meumann and others that the estimate of small time-intervals is influenced by the number of stimuli that fall within the interval. In the space illusion of sight, a single division of the standard will cause it to be underestimated, while more divisions will cause an overestimation; in touch, the effect of subdivisions depends on the absolute length of the standard.

My object, in the following experiments, was to determine the effect of single and multiple divisions of the standard on times of longer duration than those investigated by Dr. Meumann, and to discover if there existed a temporal illusion comparable to the space illusions of sight and touch.

In Dr. Meumann's investigation of time intervals, he conpares an 'empty' time - one bounded by two impressions with times 'filled' with either three, five, six, nine or twelve impressions, inclusive of the terminal stimuli. His results " are, that for times from one tenth of a second to about four seconds, when the filled time comes first, the error in estimating is constantly positive - while for longer times the error is negative.

This seems to indicate that the effect of the filling is positive for the short times, and negative for the longer. The error found by Dr. Meumann is, however, not due to the filling alone, but is the result of two factors. It is generally conceded that even when two empty times are compared, there is a similar constant error, positive for short times, negative for longer.

'See the paper by Miss Alice Robertson, on "'Geometric-Optical' Illusions in Touch" "to be published subsequently.

2 ' Beitrage zur Psychologie des Zeitbewusstseins,' Phil. Studıen, XII., p. I 27. 